SLAC-PUB-8217

August 1999

\title{
Diffuse X-ray Streaks from Defects and Surface Features in Boron Implanted Silicon
}

By U. Beck et al.

Contributed to International Conference on Solid State Spectroscopy, 5/9/99ã7/5/99, Schwaebsch Gmund Germany 


\title{
Diffuse X-ray Streaks from Defects and Surface Features
}

\section{in Boron Implanted Silicon}

\author{
U.Beck (a),C.H.Chang (b)(c),T.H.Metzger (a), and J.R.Patel(b)(c) \\ (a)Sektion Physik and CENS at Ludwig Maximilians Universitaet Muenchen \\ D-80539 Muenchen, Germany \\ (b)SSRL/SLAC, Stanford University, Stanford CA. 94309 \\ (c) ALS/LBL, 1 Cyclotron Road, Berkeley CA. \\ Corresponding author: J.R.Patel FAX: 650-926-4100 e-mail patel@ ssrl.slac.stanford.edu
}

\begin{abstract}
In a grazing incidence X-ray diffuse scattering investigation of boron implanted silicon we have discovered narrow intensity streaks along $\langle 111\rangle$ directions. From a detailed analysis of three dimensional reciprocal space maps clear evidence is found that the rodlike scattering is due to extrinsic stacking faults with an avarage diameter of $71 \mathrm{~nm}$, formed in the implanted layer after rapid thermal annealing. At the same time nanometer sized features appear at the $\mathrm{Si}$ surface which are characterized by atomic force microscopy and specular reflectivity measurements.
\end{abstract}

PACS: $68.55,68.35$ 


\section{INTRODUCTION}

The motivation for this study is the need to understand the enhanced diffusion of dopant atoms in ion implanted and annealed silicon. The dopant diffusion due to defects introduced by ion implantation can be orders of magnitude greater than bulk diffusion and limits the ability to fabricate shallow junctions for current and especially future ultra large scale integrated circuit transistors. A thorough understanding and characterization of the point defects and point defects clusters introduced by the implantation and subsequent annealing is crucial for understanding and perhaps controlling what is referred to as the phenomenon of Transient Enhanced Diffusion (TED) [1]. Here X-ray diffuse scattering at grazing incidence has been applied to study these defects.

\section{EXPERIMENTAL}

It is well known that the x-ray diffuse scattering [2] in the Bragg tails (Huang scattering) and the weak diffuse intensity [3] in between Bragg peaks ("Zwischenreflexstreuung”) can give direct information on point defects and point defect clusters. In the present study we have employed x-ray diffuse scattering close to the Bragg peak to study the formation of defects introduced after annealing ion implanted silicon. The crystal used in our studies was prepared as follows. Floating zone dislocation-free silicon crystals were cleaned and implanted with a high Boron dose at $32 \mathrm{keV}$. The wafers went through Rapid Thermal Annealing (RTA) for $10 \mathrm{sec}$ at $1070{ }^{\circ} \mathrm{C}$ in order to dissolve the implant damage and to locate the Boron predominantly in substitutional sites.

Since the implanted layers extend about $2000 \AA$ from the crystal surface we use near surface sensitive grazing incidence geometry to measure the diffuse $\mathrm{x}$-ray scattering 
around the Bragg peak in three dimensional reciprocal space. The grazing incidence arrangement [4] restricts the x-ray penetration and suppresses bulk scattering. The diffracting planes are normal to the (001) crystal surface. Both radial and angular scans were taken around the (220) Bragg peak using a position sensitive detector for efficient data collection in the region around the Bragg peak. Besides the implanted specimens the x-ray diffuse scattering was also measured for pure untreated floating zone silicon. The comparatively small thermal diffuse background scattering from the untreated crystal was subtracted from the implanted samples before analyzing the defect induced diffuse scattering .

\section{DIFFUSE SCATTERING AROUND BRAGG PEAKS IN GRAZING INCIDENCE.}

The intensity contours of diffuse scattering parallel to the surface plane around the (220) Bragg peak are shown in Fig 1 for a crystal implanted with a boron dose of $6 \mathrm{E} 15 \mathrm{~cm}^{-2}$ and annealed at $1070^{\circ} \mathrm{C}$ for 10 seconds. Besides the expected diffuse scattering close to the (220) reflection, we notice a rather unusual feature. There are lobes of intensity displaced from the Bragg peak in the [110] and [-1-10] directions. This result was obtained at a fixed value of vertical momentum transfer $\mathbf{q}_{z}=0.0587 \AA^{-1}$. The corresponding angle of incidence and exit, $\alpha_{i}$ and $\alpha_{i}$, were set to 0.37 and 0.32 degrees respectively. The angle for total external reflection for the wavelength used (1.54 $\AA$ ) amounts to $\alpha_{c}=0.22^{\circ}$. These satellite intensity lobes represent cross sections of intensity rods along [111] directions. When the $\mathrm{q}_{\|}-\mathrm{q}_{z}$ plane is mapped by varying the inplane angles in a $\theta-2 \theta$ scan and measuring the intensity along $\mathrm{q}_{\mathrm{z}}$, rod like intensity streaks were found as shown in figure 2. From the angle between the in-plane [110] and the streak direction $\left(\beta=35.3^{\circ}\right)$, we conclude that the intensity rod points in [111]. The 
obvious asymmetry in the intensity on the high angle as compared to the low angle side will be explained elsewhere. The inset in Fig. 2 shows a $q_{z}$ intensity cut through reciprocal space as indicated, demonstrating the narrow width of the streak. The full line in the inset is a fit to the data points and will be discussed in another publication. In order to analyze this unexpected feature in more detail, we have investigated the dependence of the streak intensity as a function of $\mathrm{q}_{111}$. Fig. 3 shows that over a wide range a $\mathrm{q}^{-2}$ law is found which is expected for Huang scattering. The streak intensity distribution [3,5] can be fully explained by the presence of extrinsic stacking faults on $\{111\}$ planes with a Burger vector $1 / 3 \mathrm{a}[111]$. The atomic displacements around such a stacking fault point mainly in [111] direction. These displacements are quite weak and thus Huang scattering can be expected up to large deviations q from the reciprocal lattice point where it finally changes to a Stokes-Wilson $\mathrm{q}^{-4}$ law (see Fig.3). The width of the intensity distribution perpendicular to the rods reflects directly the size of the stacking faults. From a fit (see inset in Fig.2) using the shape function of a disk (Bessel function) we obtain a stacking fault diameter of $71 \mathrm{~nm}$. This is roughly in the range of stacking fault sizes observed in cross-section TEM on a similar sample.

Finally, we have investigated the implanted Si surface by atomic force microscopy after the RTA treatment. Fig. 4a shows the dome like structures with a diameter and height of about $3.5 \mathrm{~nm}$, which are not present if the pure Si material is RTA treated in the same way. We have performed x-ray specular reflectivity measurements to study the surface density profile. In Fig. 4b the experimental results are shown together with a fit using the Parrat formalism and the density profile given in the inset. Averaging over the 
nanostructures shown in Fig. 4a the surface layer can be approximated by a density gradient, with decreasing density as the surface is approached and a thickness of $2.5 \mathrm{~nm}$.

\section{SUMMARY AND CONCLUSIONS}

In the design and fabrication of modern integrated circuits extensive use is made of computer codes both in the design of circuits and the processes necessary to implement these designs in silicon [6]. Since ion implantation is ubiquitous in the fabrication of basic transistor structures, it is important that defects and dopant motion introduced by ion implantation be thoroughly understood. Much of our understanding of the implantation process has been derived from simulation studies $[7,8]$ and modern improvements in computer codes and simulations utilizing the fast processor speeds of large computers. The simulations however need to be referenced to experiments in order to enhance the reliability of computer codes. Many ingeniously designed experiments have yielded a valuable body of data on Boron motion [9] during TED. The role of defects individual and clustered has proved more elusive. Through our studies of diffuse scattering from faults and Huang scattering from clusters of point defects, we aim to provide not only a basic understanding but quantitative data that can be used with confidence in modern computer codes. This study has enhanced our understanding of diffuse scattering from Boron implanted and annealed silicon. It remains to extend our observations to the kinetics of stacking fault and cluster growth to provide new experimental information on point defect and cluster growth and the stability of stacking fault growth and dissolution. Such detailed studies are already under way. 


\section{ACKNOWLEDGEMENTS}

We would like to acknowledge acknowledge financial support from grants supplied by NATO and Deutsche Forschungs Gemeinschaft (DPG). This work was performed at DESY, SSRL and ESRF. We are grateful to the staffs at these facilities for their unfailing patience and help. In addition one of us (JRP) would like to thank John Carruthers at INTEL for initially suggesting the problem of TED and for his encouragement and help.

\section{REFERENCES}

[1] A.E.Michel, W.Rausch, P.A.Ronsheim and R.H.Kastl, Appl. Phys. Lett., 50, 416

[2] P.H.Dederichs, J.Phys.F : Metal. Phys., 3, 471 (1973)

[3] P. Erhart, J. Nucl. Mater. 216, 170 (1994)

[4] G.Wallner, E.Burkel, H. Metzger and J.Peisl, Phys. Stat.Solidi (a) 108, 129 (1988)

[5] B.C.Larson, J.Appl.Cryst., 8, 150 (1975)

[6] M.D.Giles, S.Yu, H. W. Kennel, P. A. Packan, Mat. Res. Symp. Proc. Vol. 469, 253 (1997)

[7] G.H.Gilmer, T. Diaz de la Rubia, D.M.Stock and M.Jaraiz, Nucl.Instr \& Metods in Phys. Res. B 102, 247 (1995)

[8] J.Zhu, T. Diaz de la Rubia, L. H. Yang, C. Mailhot, and G. H. Gilmer, Phys. Rev. B 54, 4741 (1996)

[9] H. J. Gossmann,Nucl.Instr \& Meth. In Phys. Res., B 96, 187 (1996) 


\section{FIGURE CAPTIONS}

Fig.1: Topology of the diffuse intensity distribution in the (hk) plane close to the (220) Si reflection at constant vertical momentum transfer. Besides the diffuse scattering in the vicinity of the (220), two lobes of intensity appear along the radial [110] direction.

Fig.2: Diffuse scattering intensity map in the $\mathrm{q}_{\text {radial }}-\mathrm{q}_{z}$ plane. Rod like intensity streak are found along the [111] and [-1-11] directions. The inset shows an intensity ,slice“ along $\mathrm{q}_{z}$ demonstrating the width of the streak together with model calculations for extrinsic stacking faults (solid line in the inset).

Fig. 3: Diffuse scattering intensity as a function of the deviation $\mathrm{q}_{<111}$ from the (220) reflection. The $\mathrm{q}^{-2}$ law indicates Huang Scattering, the $\mathrm{q}^{-4}$ law is expected for Stokes Wilson scattering.

Fig.4: a) Atomic force microscopy image of the sample surface after boron implantation and RTA treatment. The three structural features have a diameter and height of about $3.5 \mathrm{~nm}$. b) Specular reflectivity measurement together with the fit to the data using the density model given in the inset. 


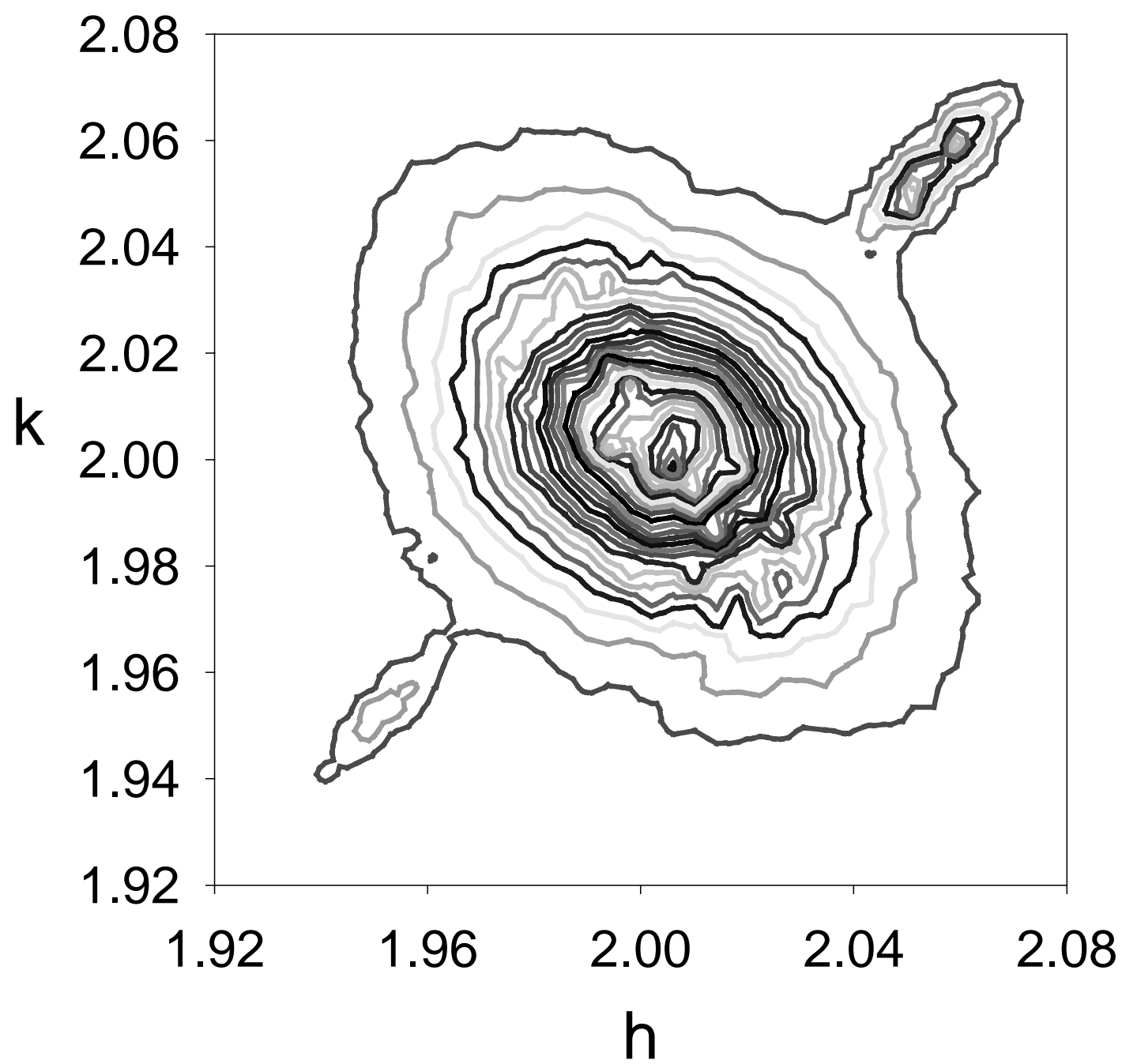

Fig. 1 Beck et al 


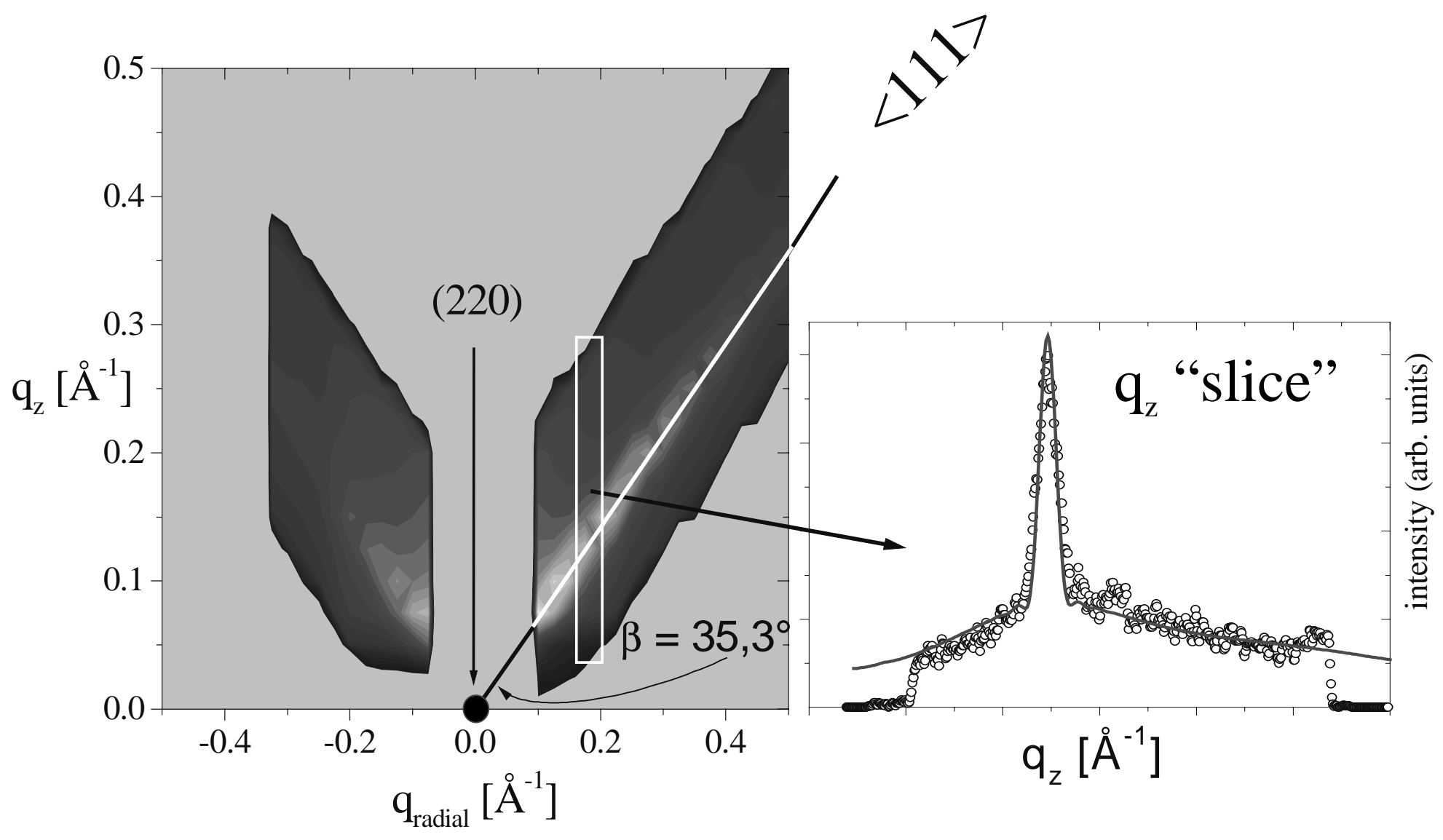

Fig. 2 Beck et al. 


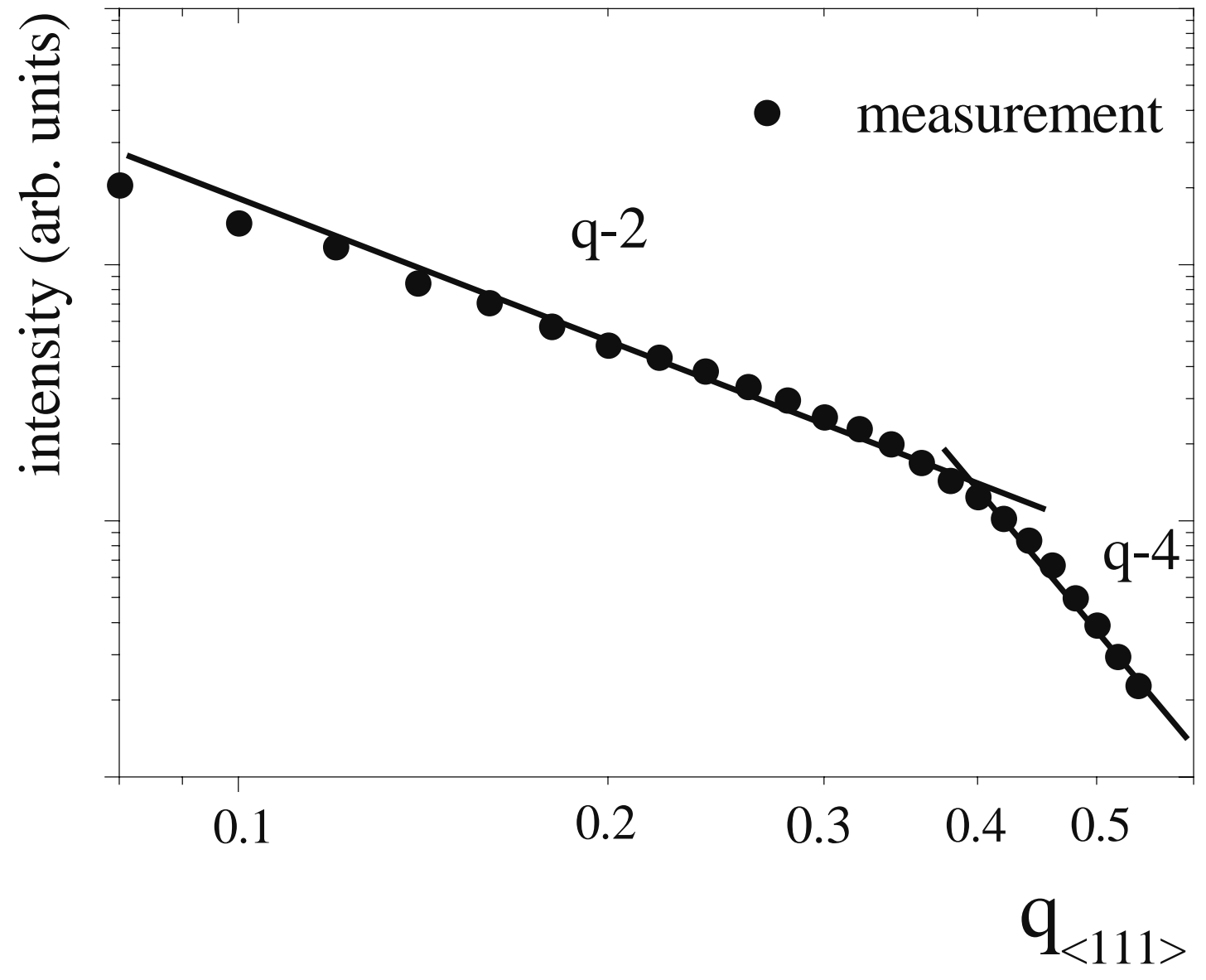

Fig.3 Beck et al. 

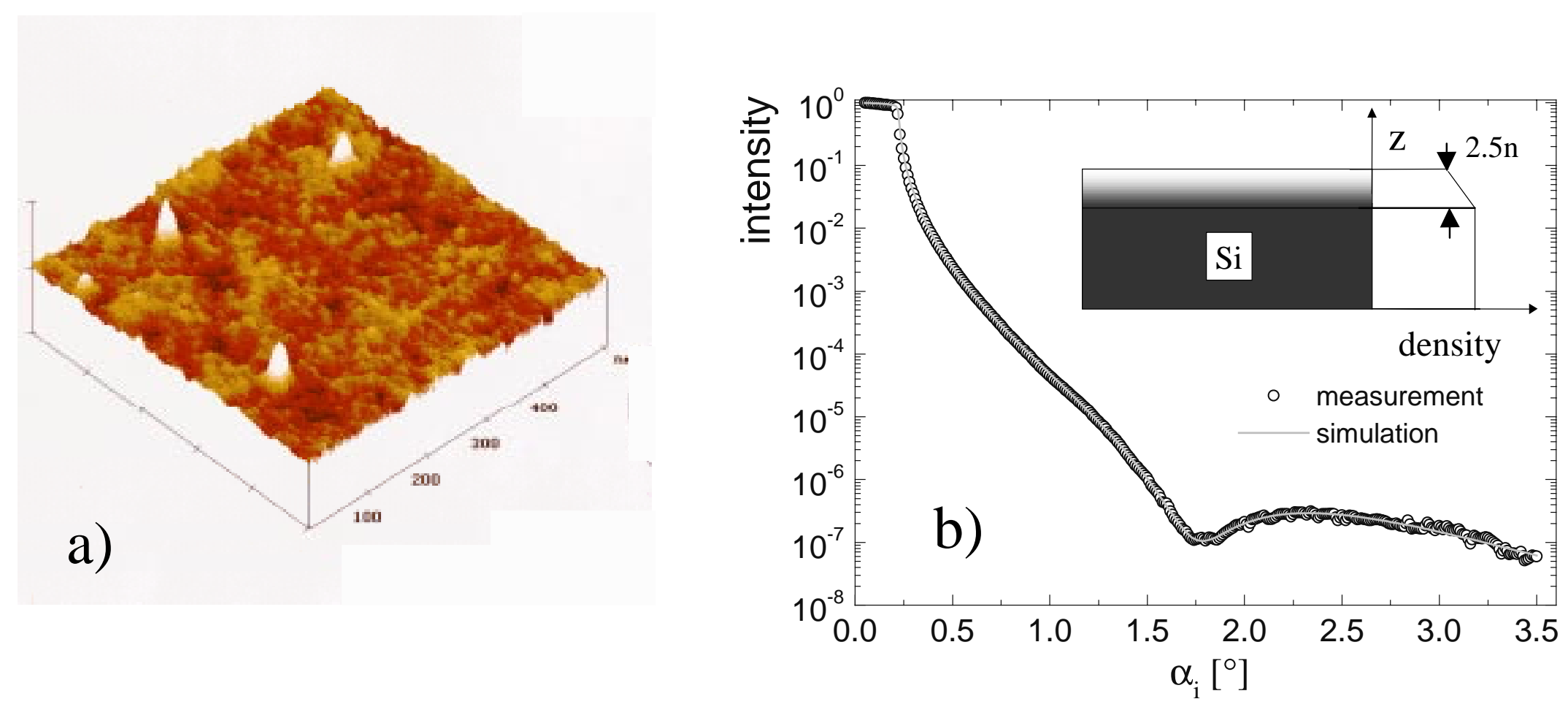

Fig.4 Beck et al. 